\title{
The hypoxia conditioned mesenchymal stem cells promote hepatocellular carcinoma progression through YAP mediated lipogenesis reprogramming
}

\author{
Yang Liu ${ }^{\dagger}$, Haozhen Ren ${ }^{\dagger}$, Yuan Zhou ${ }^{\dagger}$, Longcheng Shang, Yuheng Zhang, Faji Yang and Xiaolei Shi
}

\begin{abstract}
Background: Tumor microenvironment (TME) plays a very important role in cancer progression. The mesenchymal stem cells (MSC), a major compartment of TME, have been shown to promote hepatocellular carcinoma (HCC) progression and metastasis. As hypoxia is a common feature of TME, it is essential to investigate the effects of hypoxia on MSC during HCC progression.

Methods: The effects of hypoxia on MSC mediated cell proliferation and HCC progression were measured by cell counting kit-8 (CCK-8) assay, Edu incorporation assay and xenograft model. The role of cyclooxygenase 2 (COX2) during this process was evaluated via lentivirus mediated COX2 knockdown in MSC. We also assessed the levels and localization of yes-associated protein (YAP) in HCC cells by immunofluorescence, western blot and real-time $P C R$, in order to detect the alterations of Hippo pathway. The changes in lipogenesis was examined by triacylglycerol (TG) levels, BODIPY staining of neutral lipid, and lipogenic enzyme levels. The alterations in AKT/mTOR/SREBP1 pathway were measured by western blot. In addition, to evaluate the role of prostaglandin E receptor 4 (EP4) in MSC mediated cell proliferation under hypoxia, we manipulated the levels of EP4 in HCC cells via small interfering RNA (siRNA), EP4 antagonist or agonist.
\end{abstract}

Results: We found that MSC under hypoxia condition (hypo-MSC) could promote proliferation of HCC cell lines and tumor growth in xenograft model. Hypoxia increased COX2 expression in MSC and promoted the secretion of prostaglandin $\mathrm{E}_{2}\left(\mathrm{PGE}_{2}\right)$, which then activated YAP in HCC cells and led to increased cell proliferation. Meanwhile, YAP activation enhanced lipogenesis in HCC cell lines by upregulating AKT/mTOR/SREBP1 pathway. Knockdown or overexpression of YAP significantly decreased or increased lipogenesis. Finally, EP4 was found to mediate the effects of hypo-MSC on YAP activation and lipogenesis of HCC cells.

Conclusions: Hypo-MSC can promote HCC progression by activating YAP and the YAP mediated lipogenesis through COX2/PGE $/$ EP4 axis. The communication between MSC and cancer cells may be a potential therapeutic target for inhibiting cancer growth.

Keywords: Hypoxia, Mesenchymal stem cells, YAP, Lipogenesis

\footnotetext{
* Correspondence: njsxl2000@163.coml

†Yang Liu, Haozhen Ren and Yuan Zhou contributed equally to this work. Department of Hepatobiliary Surgery, The Affiliated Drum Tower Hospital of Nanjing University Medical School, NO.321 Zhongshan Road, Nanjing, Jiangsu 210008, People's Republic of China
} 


\section{Background}

Recently, the communication between tumor microenvironment (TME) and cancer cells has been increasingly appreciated as a pivotal contributor to tumor progression $[1,2]$. One important component of TME is the mesenchymal stem cells (MSC), which are multipotent cells that reside in various tissues, and get recruited to primary tumor site from the bone marrow in response to tumor derived soluble factors [1,3]. The link between MSC and tumor progression was established by the promoting effects from MSC on tumor growth and metastasis in mouse lymphoma, melanoma and breast cancer models when injected together with tumor cells. This effect is mainly mediated by the numerous factors and chemokines produced by MSC, such as transforming growth factor- $\beta$ (TGF $\beta), \quad$ CC-chemokine ligand 5 (CCL5), CXC-chemokine ligand 10 (CXCL10) and CXCL12 [1]. A characteristic phenomenon of TME in all solid tumor types, including hepatocellular carcinoma (HCC), is hypoxia, which has been found to promote angiogenesis, metabolic reprogramming, extracellular matrix remodeling and epithelial-mesenchymal transition (EMT) [4]. However, the role of MSC in HCC progression, especially under the hypoxia condition, has not been fully investigated.

Yes-associated protein (YAP) is the central transcriptional co-activator of Hippo pathway. It controls the trans-activation of a variety of target genes to regulate organ size, promote cell proliferation and inhibit apoptosis [5]. Recent studies confirmed the role of YAP in HCC progression, and found that YAP activity was increased during the early development of liver cancer [6, 7]. Moreover, liver-specific overexpression of YAP leads to hepatomegaly and subsequent tumor formation $[8,9]$.

Recently, altered lipid metabolism has been recognized as a hallmark of cancer [10]. Continuous de novo lipogenesis provides cancer cells with membrane building blocks, signaling lipid molecules and post-translational protein modifications to support rapid cell proliferation [11]. Increased lipid biosynthesis has been found to promote HCC development; moreover, the suppression of fatty acid synthase (FASN), a rate-limiting enzyme in lipogenesis, could impair the growth of human HCC cells [12]. Emerging evidences suggest that YAP can coordinate nutrient availability with cell growth and tissue homeostasis $[13,14]$. Also, YAP was found to participate in metabolism regulation, such as glycolysis and lipogenesis [12, 13], in order to promote HCC progression. Therefore, the detailed mechanisms of how lipid metabolism is altered in HCC and how it is the related to YAP activation would be important questions to address.

In this study, we found that the MSC under hypoxia condition could promote HCC progression. More specifically, we found that hypoxia increased cyclooxygenase 2
(COX2) expression in MSC, leading to enhanced secretion of prostaglandin $\mathrm{E}_{2}\left(\mathrm{PGE}_{2}\right)$. Furthermore, the $\mathrm{PGE}_{2}$ secreted by MSC activated YAP in HCC cells, which then increased lipogenesis and promoted cell proliferation.

\section{Methods \\ Cell lines and cell culture}

HCC cell lines 7402 and Hep3b were purchased from the Shanghai Institutes for Biological Sciences. HCC cell line 7402 was cultured in RPMI 1640 medium (Corning Inc., Corning, NY, USA), and Hep3b was cultured in MEM medium (Corning Inc.). Both mediums were supplemented with $10 \%$ fetal calf serum (FBS), $100 \mathrm{U} / \mathrm{ml}$ penicillin and $100 \mathrm{~g} / \mathrm{ml}$ streptomycin. Cells were maintained in a humidified incubator with $5 \% \mathrm{CO}_{2}$ at $37^{\circ} \mathrm{C}$. Human umbilical MSC were purchased from ScienCell (San Diego, California, USA) and cultured in DMEM/ F-12 medium (Corning Inc.). MSC at passage 3-10 were used for experiments. When MSC grew to about $80 \%$ confluence, they were switched to serum-free medium and cultured for $48 \mathrm{~h}$ at $37^{\circ} \mathrm{C}$ under hypoxic $\left(1.5 \% \mathrm{O}_{2}\right)$ (hypo-MSC) or normoxic $\left(21.0 \% \mathrm{O}_{2}\right)$ conditions. The condition medium (CM) was harvested, purified by centrifugation and frozen at $-80^{\circ} \mathrm{C}$ for further experiment.

\section{COX2 knockdown in MSC and YAP overexpression in HCC cell lines}

To inhibit COX2 in MSC, lentivirus carrying shRNA against COX2 (GeneChem, Shanghai, China) was used to infect MSC [named MSC-COX2(-)]. The PGE 2 levels in supernatant was detected by ELISA (enzyme-linked immunosorbent assay) kit (R\&D System, Minneapolis, Minnesota, USA). To overexpress YAP in HCC cell lines, cells were infected with lentivirus carrying YAP gene (GeneChem). The sequences for the COX2 shRNA and YAP gene were listed in Additional file 1: Table S4.

\section{Reagents \\ $\mathrm{PGE}_{2}, \mathrm{CAY} 10598$ (EP4 agonist) and GW627368X (EP4 inhibitor) were bought from Cayman Chemical (Ann Arbor, MI, USA). AKT inhibitor, LY294002 was bought from Selleck Chemicals (Houston, TX, USA), SREBP1 inhibitor, Fatostatin was bought from MedChemExpress (MCE, Monmouth Junction, NJ, USA).}

\section{Western blot and immunoprecipitation (IP)}

Proteins were extracted from cells using RIPA buffer (KeyGEN BioTECH, Nanjing, China) with phenylmethylsulfonyl fluoride (PMSF) at $4{ }^{\circ} \mathrm{C}$ for $30 \mathrm{~min}$. Western blot and IP were performed as previously described $[15,16]$. Primary antibodies were listed in Additional file 1: Table S1. 
RNA isolation and quantitative real-time PCR (qRT-PCR) Total RNA was isolated from HCC cells by TRIzol, followed by real-time PCR analysis with ABI 7500 (Applied Biosystems, Foster City, CA, USA). $18 \mathrm{~s}$ was used as an internal control. The $2^{-\Delta \Delta C T}$ method was employed to determine the relative mRNA expression. The primer sequences were listed in Additional file 1: Table S2.

\section{Cell viability assay}

Seven thousand four hundred two and Hep3b cells were plated in 96-well plates at the density of $2 \times 10^{3} /$ well and cultured overnight. On the next day, their medium was replaced with CM $(100 \mu \mathrm{L} /$ well $)$ or fresh medium supplemented with $\mathrm{PGE}_{2}$. Cell viability was determined by cell counting kit-8 (CCK-8) assay (MCE).

\section{Small interfering RNA (siRNA) and transfection}

siRNAs against YAP and prostaglandin $\mathrm{E}$ receptor 4 (EP4), as well as control siRNAs were obtained from RiboBio (GuangZhou, China). The detailed sequences of siRNAs were listed in Additional file 1: Table S3. Transfection with siRNAs and miRNAs were completed using riboFECT $^{\text {тм }}$ CP (RiboBio) according to the manufacturer's instruction.

\section{Quantification of neutral lipid and triacylglycerol (TG)}

The lipophilic fluorescence dye BODIPY 493/503 (Invitrogen, ThermoFisher Scientific, Eugene, OR, USA) was used to monitor the content of neutral lipids in HCC cells as previously described [17]. The TG in cells and tissues were measured by EnzyChrom ${ }^{\mathrm{T}}{ }^{\text {Triglyceride }}$ Assay Kits (BioAssay Systems, Hayward, CA, USA) following manufacturer's protocol.

\section{Immunofluorescence staining}

HCC cells were seeded in 24-well cell culture cluster. After treatment, cells were fixed with $4 \%$ paraformaldehyde for $20 \mathrm{~min}$, permeabilized with $0.5 \%$ Triton X-100 in PBS at room temperature for $15 \mathrm{~min}$, and then blocked with 10\% bovine serum albumin in PBS for $1 \mathrm{~h}$. Subsequently, cells were incubated with primary antibodies against YAP (1:100, Abcam, Cambridge, MA, UK) or SREBP1(1:50, Santa Cruz Biotechnology, Dallas, TX, USA) at $4{ }^{\circ} \mathrm{C}$ overnight, followed by incubation with goat anti-rabbit or anti-mouse IgG H\&L antibodies (Alexa Fluor $\left.{ }^{\oplus} 488\right)$ (1200, Abcam) for $1 \mathrm{~h}$. Nuclei were counterstained with 4',6-diamidino-2-phenylindole (DAPI, KeyGEN BioTECH) and images were captured using fluorescence microscopy.

\section{Edu assay}

Cells were seeded on glass coverslips in 24 well plates. After treatment, cells were incubated with Edu (Beyotime Biotechnology, Shanghai, China) for $2 \mathrm{~h}$ at $37^{\circ} \mathrm{C}$, and fixed in $4 \%$ paraformaldehyde. After permeabilization with $0.5 \%$ Triton-X, the cells were reacted with click additive solution (Beyotime Biotechnology) for $30 \mathrm{~min}$. Subsequently, the DNA contents of the cells were stained with Hoechst 33342 for $10 \mathrm{~min}$ and visualized under a fluorescence microscope. Edu positive cells were quantified from three randomly selected fields in each well, and each experiment was repeated for three times.

\section{In vivo tumorigenesis assays}

A total of $1 \times 10^{6}$ tumor cells were injected alone or co-injected with MSC $\left(2 \times 10^{5}\right)$ into 4-week-old $\mathrm{BALB} / \mathrm{c}$ nude mice subcutaneously ( $n=6$ each group). After 4 weeks, mice were sacrificed, and the tumors were harvested, weighed, and saved for further studies. All procedures were approved by the Ethics Committee for Animal Experimentation of the Affiliated Drum Tower Hospital of Nanjing University Medical School. The harvested tumors were stained with Ki-67 (Abcam), YAP (Abcam) and CD90 (Abcam) by immunohistochemistry (IHC).

\section{Statistical analysis}

All the data are expressed as mean \pm SD. Student $t$ test and one-way analysis of variance (ANOVA) were used to compare the data. $P<0.05$ was considered statistically significant.

\section{Results}

Hypo-MSC promotes the proliferation of HCC cells both in vitro and in vivo

To explore the effects of MSC under hypoxia condition (hypo-MSC) on HCC cell proliferation, we performed CCK- 8 assays and found that the CM of MSC under normoxia and hypoxia conditions could both promote HCC cells growth, but the hypo-MSC could increase cell growth more robustly (Fig. 1a). Consistently, Edu incorporation assay showed increased proliferation of HCC cells in the presence of either MSC or hypoMSC-CM (Fig. 1b, c). Next, we tested whether hypoMSC could promote HCC growth in vivo with xenograft model. Seven thousand four hundred two or Hep3b cells were subcutaneously implanted into nude mice, either alone or co-implanted with MSC or hypo-MSC. By measuring tumor mass and volume, we found that the tumors with hypo-MSC co-injection exhibited the fastest growth rate (Fig. 1d, e). Consistently, these tumors showed higher Ki-67 levels compared to other groups, as revealed by IHC staining (Fig. 1f and Additional file 2: Figure S1). Taken together, these findings demonstrated that hypo-MSC could promote HCC cell proliferation both in vitro and in vivo. 
a

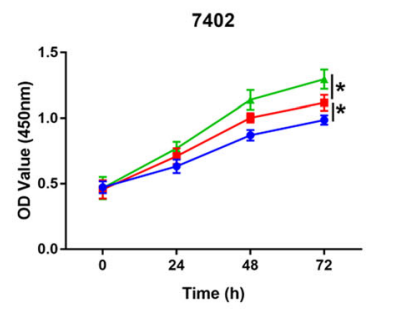

b
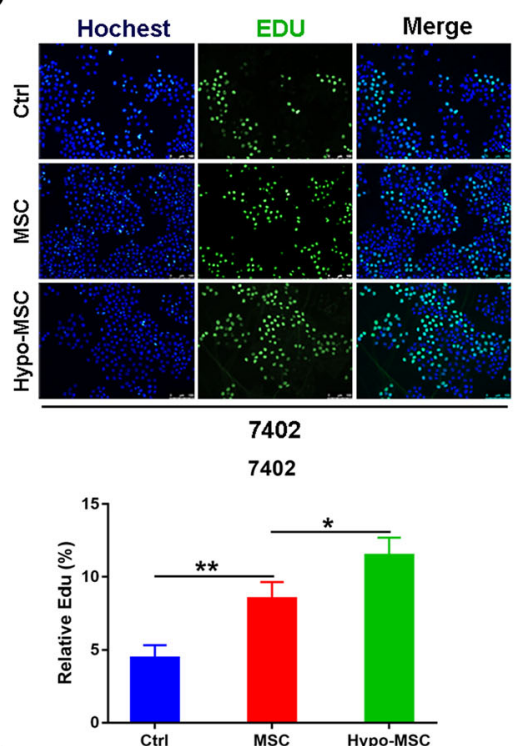

d
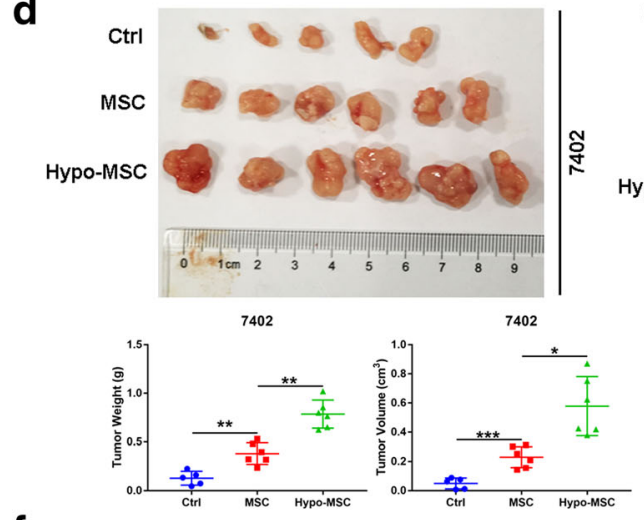

f

7402

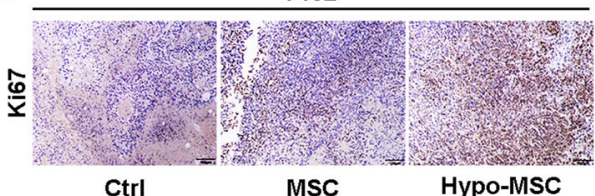

$\rightarrow$ Ctrl
- MSC

- Hypo-MSC

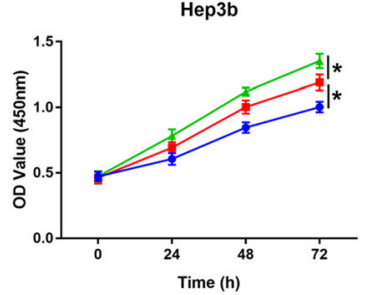

$\rightarrow$ Ctrl

- Hypo-MSC

C

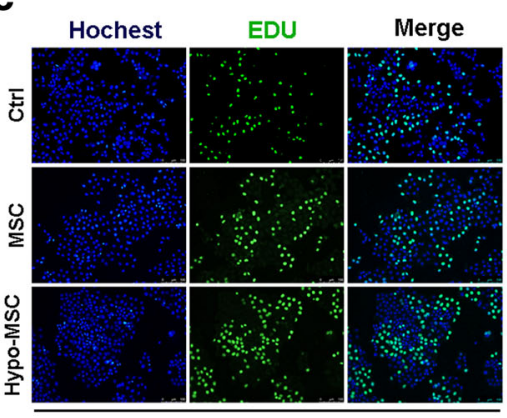

Hep3b

Hep3b

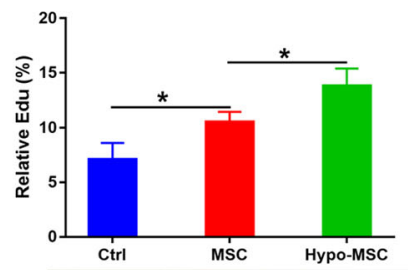

e
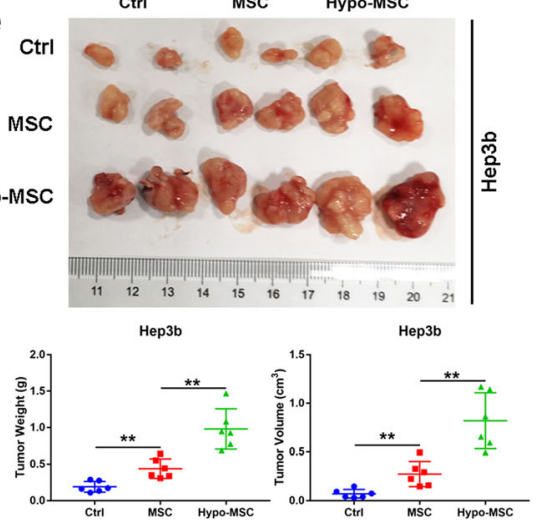

Hep3b

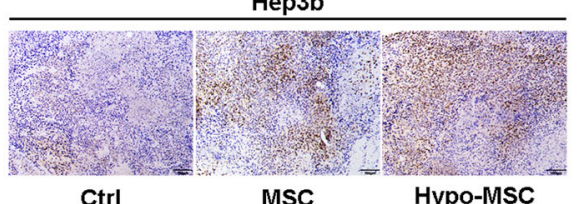

Fig. 1 Hypo-MSC promotes the proliferation of HCC cells both in vitro and in vivo. a The proliferation ability of 7402 and Hep3b under indicated conditions $(n=3)$. $\mathbf{b}$-c The representative images and quantification of Edu positive cells in 7402 and Hep3b under indicated conditions ( $n=3$ ). $\mathbf{d}$-e Images of xenograft tumors, tumor volume and weight measurement of 7402 or Hep3b injected alone or co-injected with MSC or hypo-MSC ( $n=5-6)$. $\mathbf{f}$ Representative images of Ki-67 in 7402 or Hep3b injected alone or co-injected with MSC or hypo-MSC. $\left(^{*} p<0.05\right.$, ${ }^{* *} p<0.01,{ }^{* * *} p<0.001$, hypo-MSC: hypoxia conditioned MSC) 


\section{Hypo-MSC promotes HCC progression through COX2/ $\mathrm{PGE}_{2}$ axis}

Next, we investigated the mechanism of how hypo-MSC promotes HCC progression. Previous studies have shown that hypoxia could upregulate $\operatorname{COX} 2$, which then increased $\mathrm{PGE}_{2}$ expression and promoted the growth of esophageal squamous cell carcinoma and colon cancer [18-20]. In $\mathrm{HCC}, \mathrm{COX} 2 / \mathrm{PGE}_{2}$ pathway also plays a key role in tumor progression [21]. Therefore, we investigated whether COX2 expression was increased in hypo-MSC. Indeed, hypoxia increased the expression of COX2 in MSC, and led to enhanced $\mathrm{PGE}_{2}$ secretion (Fig. 2a). To confirm the role of COX2 upregulation in MSC on HCC progression, we knocked down COX2 in MSC via lentivirus, and found that the $\mathrm{PGE}_{2}$ secretion of MSC was significantly reduced under hypoxia condition (Fig. 2b). CCK-8 and Edu incorporation assays showed that the increased cell proliferation of HCC cells was significantly suppressed by COX2 knockdown in MSC (Fig. 2c-e). However, the effect of hypo-MSC on HCC cells growth was not completely eliminated by COX2 knockdown, indicating that other mechanisms might be involved (Fig. 2c-e). Consistently, exogenous $\mathrm{PGE}_{2}$ could promote $\mathrm{HCC}$ cell growth in a dose dependent manner (Additional file 3: Figure S2). Furthermore, we tested the effects of COX2 knockdown in xenograft models. Both tumor mass and cancer cell proliferation, revealed by Ki-67 staining, were significantly decreased when COX2 was knocked down in MSC (Fig. 2f-g and Additional file 2: Figure S1). Overall, we found that hypoxia led to upregulated COX2 expression in MSC, which promoted $\mathrm{HCC}$ progression.

\section{Hypo-MSC promotes HCC progression through YAP activation}

YAP plays an important role in the development of HCC [6]. Recent studies have revealed the relationships among COX2, COX2-derived $\mathrm{PGE}_{2}$ secretion and YAP activation in cancer development [22-24]. Therefore, we next investigated whether hypo-MSC could also regulate YAP activity in HCC. Western blot showed that YAP was upregulated in $\mathrm{HCC}$ cells when the cells were treated with MSC or hypo-MSC-CM (Fig. 3a). Consistently, the mRNA levels of YAP and YAP target genes, CTGF and CYR61, were also upregulated in HCC cells by hypo-MSC treatment (Fig. 3b). Immunofluorescence staining confirmed that the translocation of YAP into nucleus in hypo-MSC treated HCC cells (Fig. 3c, d), which was correlated with YAP phosphorylation level (Fig. 3a), as YAP phosphorylation at Ser127 induces its cytoplasmic retention and subsequent degradation [9]. Conversely, knockdown of COX2 in MSC reversed YAP expression and nuclear translocation in HCC cells, even under hypoxia condition (Fig. 3a-d). Consistently, exogenous $\mathrm{PGE}_{2}$ showed the similar effect on YAP activation Additional file 4: Figure S3. Moreover, the xenograft tumors showed YAP activation in groups with hypo-MSC co-injection, as indicated by IHC staining (Fig. 3e). Since hypo-MSC could activate YAP in HCC cell lines, we next asked whether YAP was required for hypo-MSC induced cell proliferation. We knocked down YAP in HCC cell lines with siRNA, and found that the increased HCC cell proliferation mediated by hypo-MSC was abolished by YAP knockdown (Fig. 3f). Edu incorporation assays further confirmed this result (Fig. 3g). Collectively, our results suggested that hypo-MSC could activate YAP in HCC cells, leading to increased cell proliferation.

\section{Hypo-MSC promotes HCC progression through YAP mediated lipogenesis}

Altered lipid metabolism affects numerous cellular processes, including proliferation, motility, and tumorigenesis [10]. Therefore, to examine whether hypo-MSC can affect lipid metabolism in HCC cells, we evaluated the lipid contents in $\mathrm{HCC}$ cell lines when treated with hypo-MSC. Indeed, the intracellular levels of TG were significantly increased in HCC cells when treated with hypo-MSC (Fig. 4a), which was further supported by BODIPY 493/503 staining, suggesting that MSC or hypo-MSC could increase the levels of intracellular neutral lipids in HCC cells (Fig. 4b). Moreover, to test the role of MSC in regulating de novo fatty acid synthesis, we analyzed the expression levels of key lipogenic enzymes in HCC cells, including ATP citrate lyase (ACLY), Acetyl-CoA carboxylase (ACC1), FASN, and Stearoyl-CoA desaturase 1 (SCD1). The results showed that all these lipogenic enzymes were significantly increased in hypo-MSC treated HCC cells (Fig. 4c). Consistently, the TG levels were also increased in the xenograft tumors co-injected with hypo-MSC co-injection (Fig. 4d). Furthermore, the enhanced lipogenesis in HCC cell lines and tumor tissues could be rescued by COX2 knockdown in MSC, even under hypoxia condition.

Previous studies have shown that YAP participates in metabolism regulation, such as promoting glycolysis and lipogenesis $[10,11]$. Thus, we further explored the role of YAP on hypo-MSC induced lipogenesis. Knockdown (KD) or overexpression (OE) of YAP in HCC cells significantly decreased or increased lipogenesis (Fig. 4e-g). Also, the hypo-MSC induced lipogenesis was diminished when YAP was knocked down, as shown by intracellular TG levels, BODIPY staining intensity and lipogenic enzymes expression in HCC cells (Fig. 4h-j). Taken together, our results suggested that hypo-MSC promoted $\mathrm{HCC}$ progression through YAP mediated lipogenesis. 
a

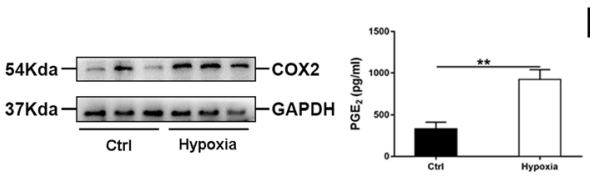

b

C

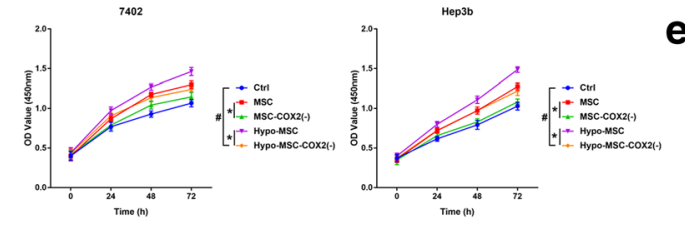

e
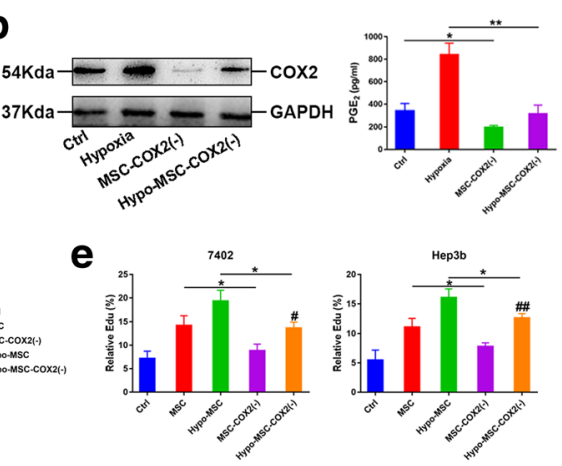

d

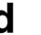

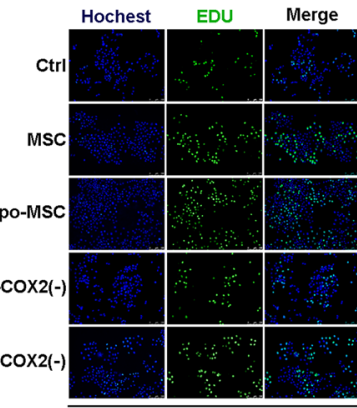

7402
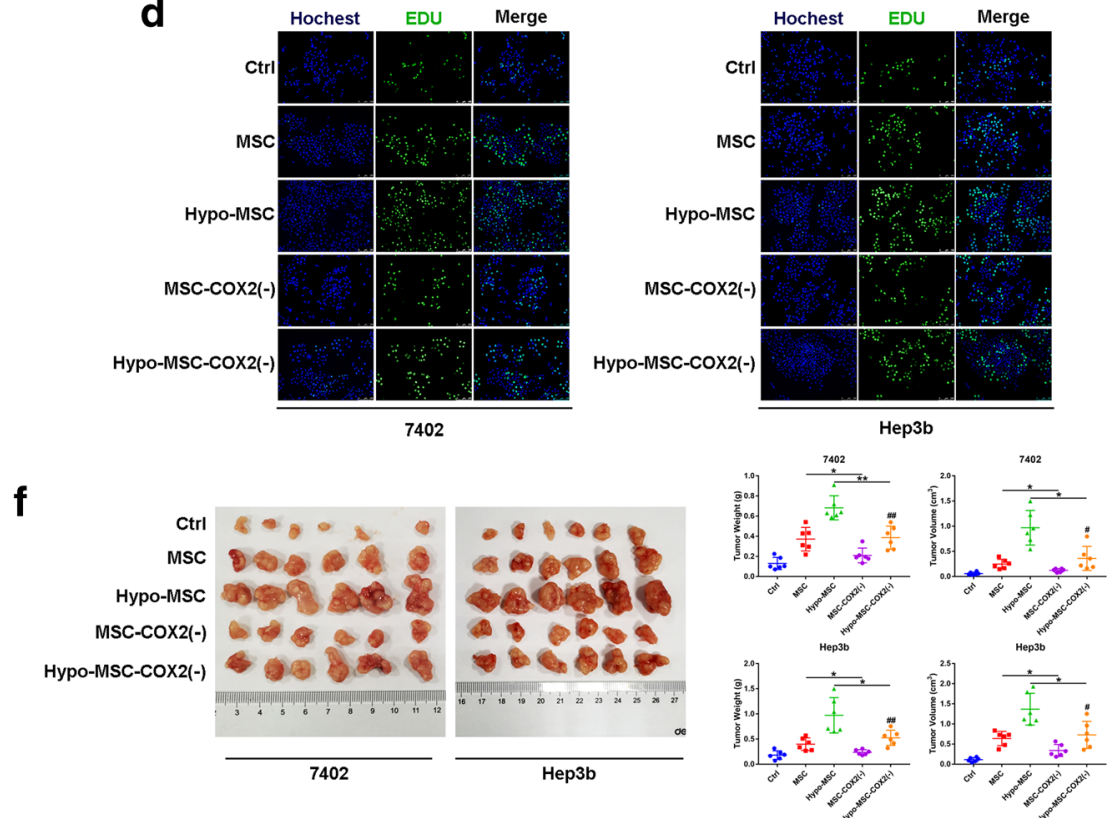

g

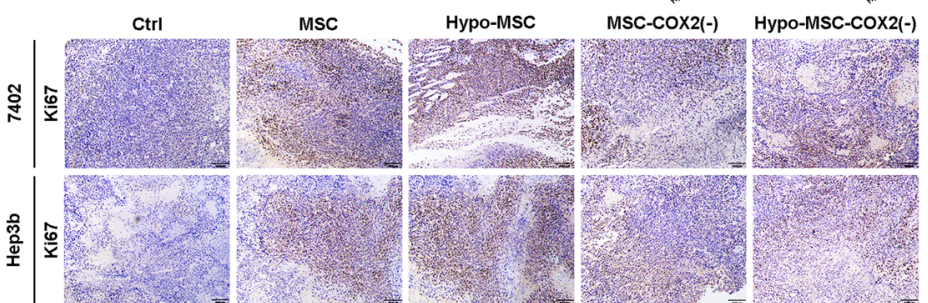

Fig. 2 Hypo-MSC promotes HCC progression through COX2/PGE 2 axis. a Protein levels of COX2 and PGE 2 secreted by MSC under normal or hypoxia condition $(n=3)$. b Protein levels of COX2 and PGE 2 secreted by MSC after COX2 knockdown by lentivirus under normal or hypoxia condition $(n=3)$. $\mathbf{c}$ The proliferation ability of 7402 and Hep3b under indicated conditions $(n=3)$. $\mathbf{d}$-e Representative images and quantification of Edu positive cells in 7402 and Hep3b under indicated conditions $(n=3)$. f Images of xenograft tumors, tumor volume and weight measurement of 7402 or Hep3b injected alone or co-injected with MSC $(n=6)$. $\mathbf{g}$ The representative images of Ki-67 of xenograft tumors in 7402 or Hep3b injected alone or co-injected with MSC. ( ${ }^{*} p<0.05,{ }^{* *} p<0.01,{ }^{\#} p<0.05$ vs Ctrl, ${ }^{\# \#} p<0.01$ vs Ctrl)

\section{YAP enhances lipogenesis via AKT-mTOR-SREBP1 pathway}

Sterol regulatory elementary binding proteins (SREBPs) are important enzymes in regulating fatty acid synthesis [25]. A recent study indicated that SREBP1 contributed to HCC progression by promoting cancer cell growth and metastasis [26]. It has also been shown that LATS2, a core member of Hippo pathway, inhibits SREBP1 and suppresses hepatic cholesterol accumulation [27]. Therefore, we then asked whether hypo-MSC increases SREBP1 expression via YAP to promote lipogenesis. Western blot analysis showed that SREBP1 was upregulated in hypo-MSC treated HCC cells, and COX2 knockdown inhibited SREBP1 expression (Fig. 5a). Moreover, immunofluorescence staining showed that the nuclear accumulation of SREBP1 was increased in hypo-MSC 
a

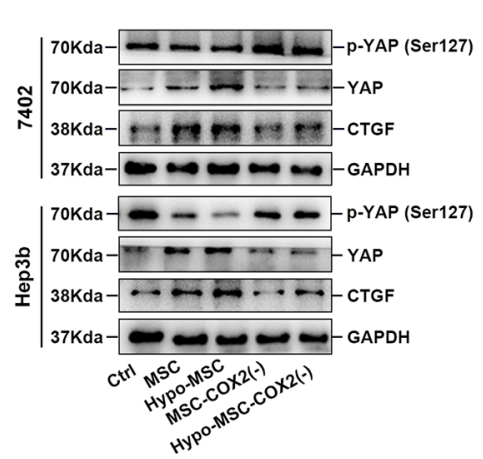

b
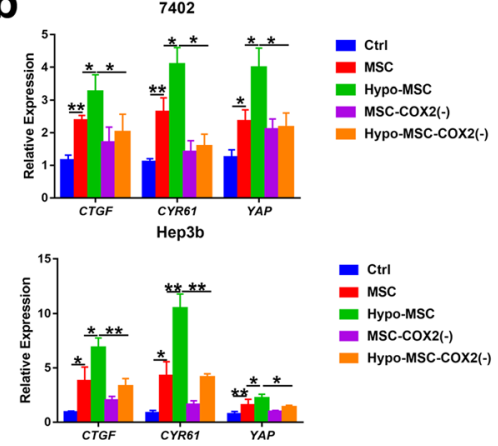

- Ctrl

Hypo-Msc

MSC-COx2(-)

Hypo-MSC-COX2(-)
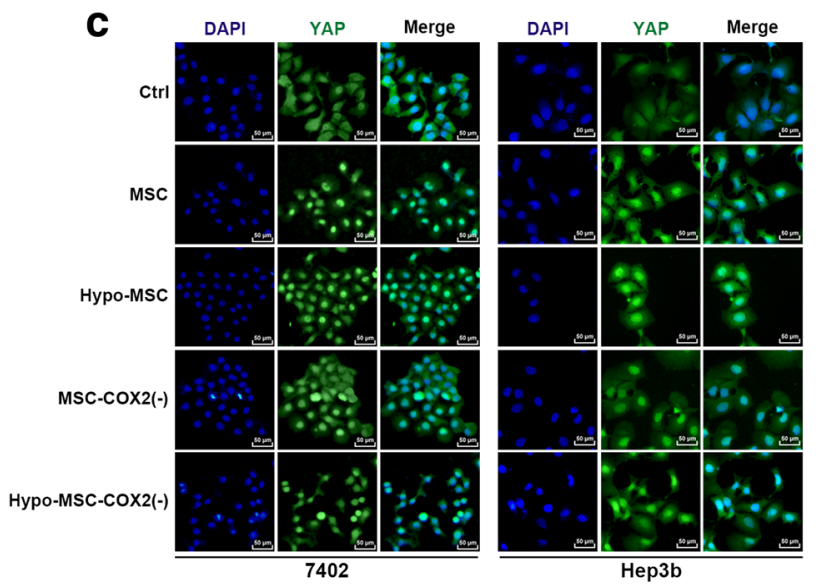

d

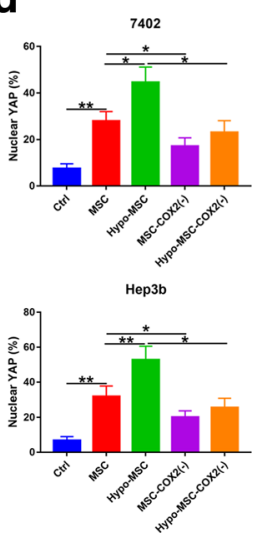

e<smiles>[Mg][Mg]</smiles>

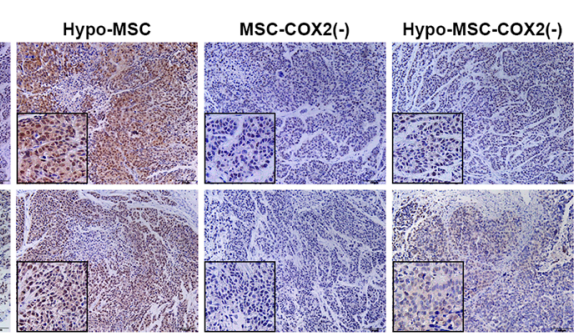

f

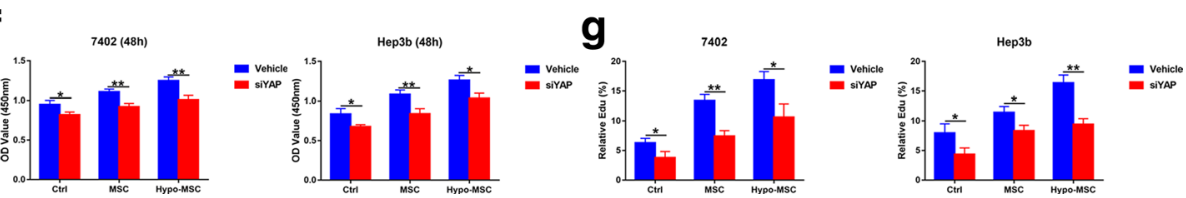

Fig. 3 Hypo-MSC activates YAP signaling to promote cell proliferation in HCC cells. a Protein levels of YAP and its target CTGF in 7402 and Hep3b under indicated conditions. b The mRNA levels of YAP and its target genes (CTGF, CYR61) in 7402 and Hep3b under indicated conditions. c-d Immunofluorescence of YAP in in 7402 and Hep3b c and quantitative data percentage of cells with nuclear YAP $(\mathbf{d}, n=3)$. e The representative images of YAP in xenograft tumors. $\mathbf{f}$ The proliferation ability of 7402 and Hep3b after YAP knockdown via siRNA under indicated conditions $(n=3)$. g Quantification of Edu positive cells in 7402 and Hep3b cells after YAP knockdown via siRNA under indicated conditions $(n=$ 3). $\left({ }^{*} p<0.05,{ }^{* *} p<0.01\right)$

treated HCC cells (Fig. 5b), indicating the active transcription of SRE-containing genes by SREBP1. Consistently, YAP KD or OE in HCC cells significantly inhibited or increased SREBP1 expression (Fig. 5c, d). To test the role of SREBP1 in HCC growth, we knocked down SREBP1 in HCC cells via siRNA, and found that both YAP and SREBP1 were necessary for HCC cells growth, as demonstrated by the CCK- 8 and Edu assays (Additional file 5: Figure S4). Furthermore, to confirm the role of SREBP1 in YAP mediated lipogenesis, we treated YAP OE cells with SREBP1 inhibitor, Fatostatin. Fatostatin treatment significantly decreased the intracellular TG levels, staining intensity, and expression of lipogenic enzymes (Additional file 6: Figure S5). These results indicated that the hypo-MSC could induce lipogenesis in HCC cells via YAP mediated SREBP1 upregulation. 


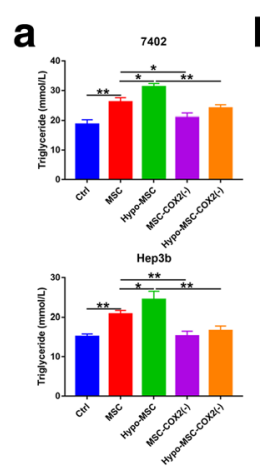

d

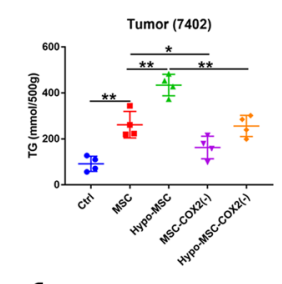

$\mathbf{f}$

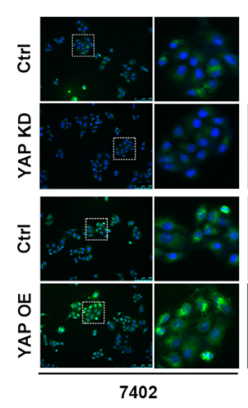

i
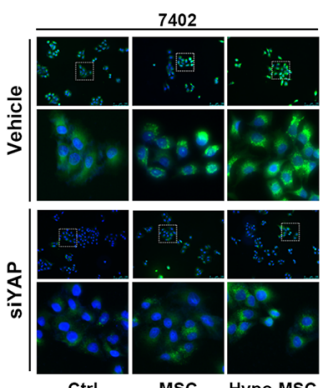
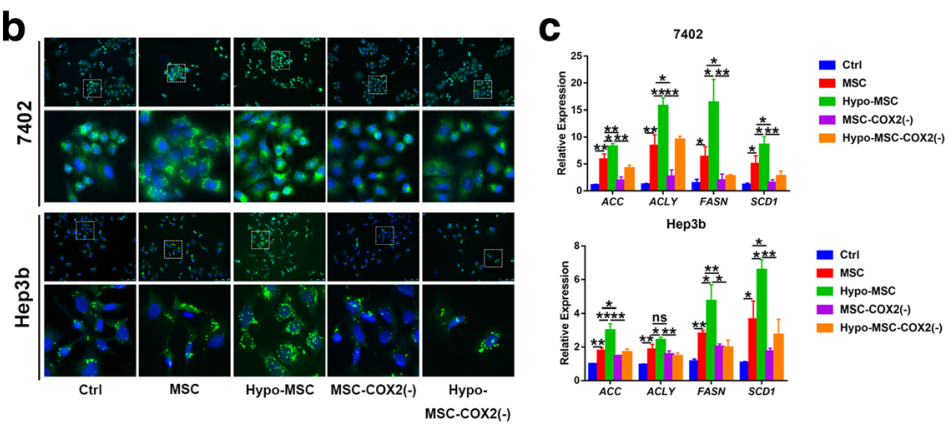

e
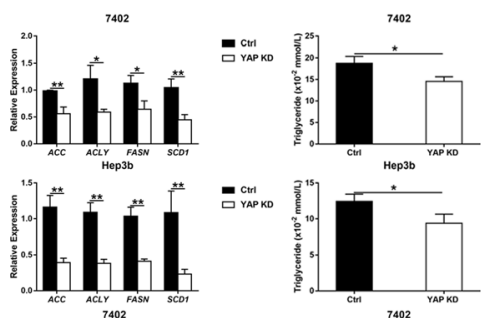

g
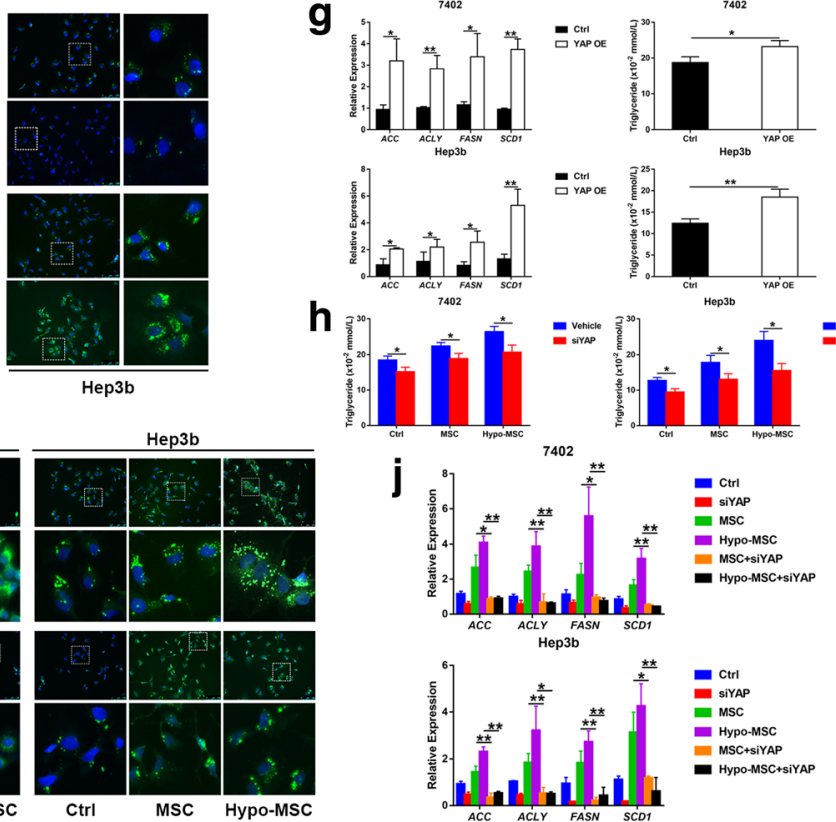

h

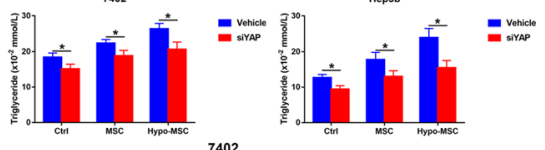

j
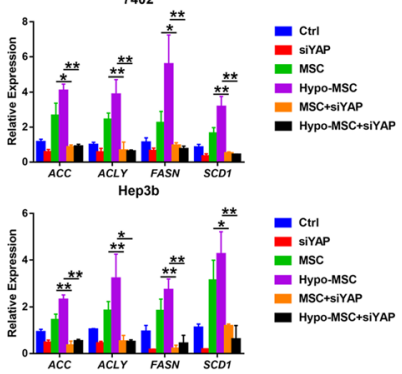

Fig. 4 Hypo-MSC enhances lipogenesis via YAP in HCC cell lines. a Cellular TG levels were detected in 7402 and Hep3b under indicated conditions $(n=3)$. b The content of neutral lipids was detected by with BODIPY 493/503 dye staining in 7402 and Hep3b under indicated conditions. c The mRNA levels of lipogenic enzymes in 7402 and Hep3b under indicated conditions ( $n=3)$. $\mathbf{d}$ Levels of TG in xenograft tumors on different groups $(n=4)$. e-g Cellular content of TG, neutral lipids and lipogenic enzymes were detected in YAP knockdown (YAP KD) or overexpression (YAP OE) cells. $\mathbf{h}$ Cellular content of TG were detected in YAP knockdown (siYAP) cells under indicated conditions ( $n=3$ ). $\mathbf{i}$ The content of neutral lipids was detected by BODIPY 493/503 dye staining in YAP knockdown (siYAP) cells under indicated conditions. $\mathbf{j}$ The mRNA levels of lipogenic enzymes in YAP knockdown (siYAP) cells under indicated conditions $(n=3) .\left({ }^{*} p<0.05,{ }^{* *} p<0.01\right)$

Next, we explored the mechanism of how YAP activated SREBP1. As YAP is a transcriptional co-activator, we performed CO-IP to see whether YAP directly binds to SREBP1. However, we did not see directed interaction between YAP and SREBP1 (Additional file 7: Figure S6). Previous studies have demonstrated that the AKT/ mTOR pathway plays an important role in regulating fatty acid synthesis [12], and mTOR can promote de novo lipid synthesis through SREBP [28]. Since increased YAP expression in human liver tumors is associated with high levels of p-AKT [29], we hypothesized that in hypoMSC treated HCC cells, YAP activation activates AKT/ mTOR pathway, which then promotes the SREBP1-mediated upregulation of lipogenic enzymes. Western blot 
a

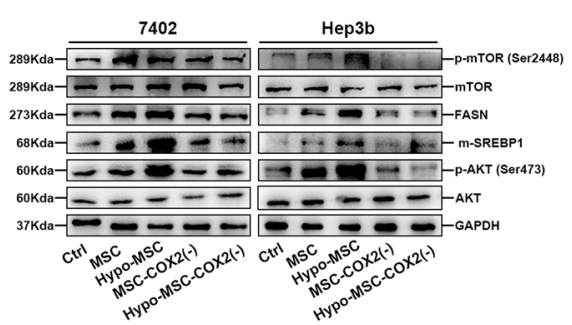

C

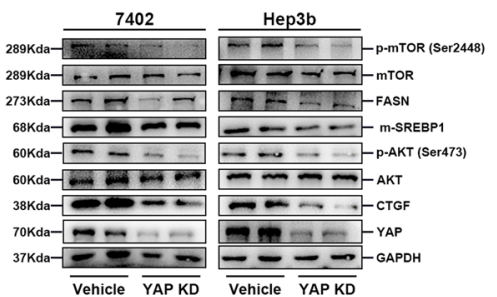

d

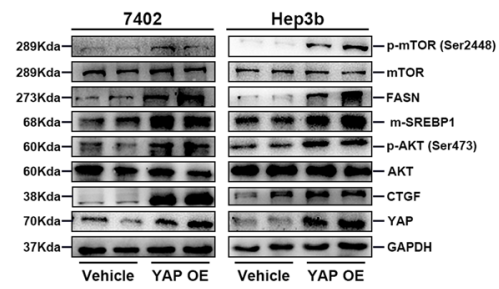

f

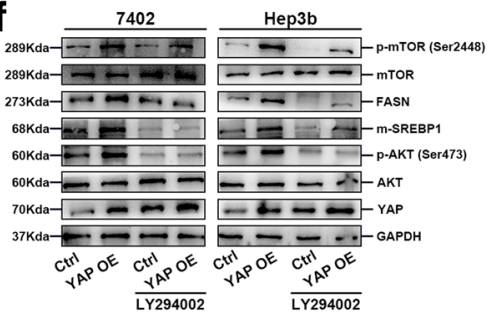

h

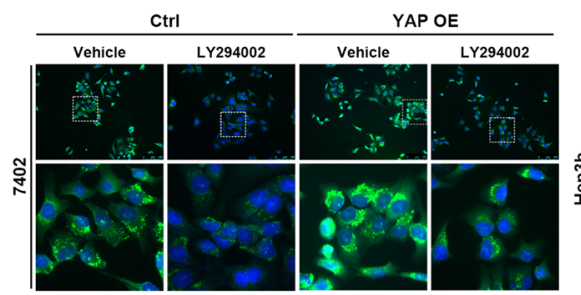

b
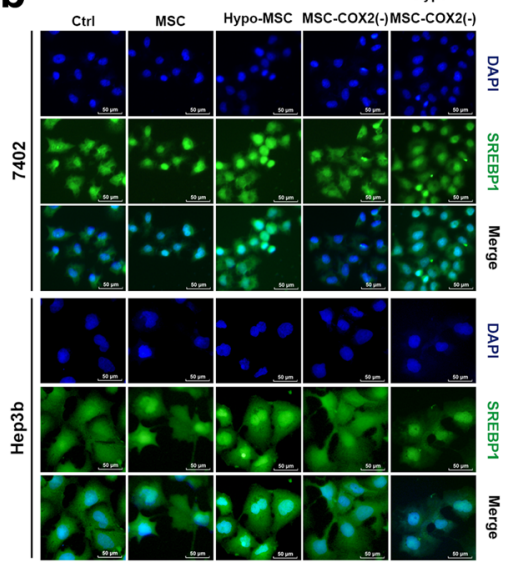

e

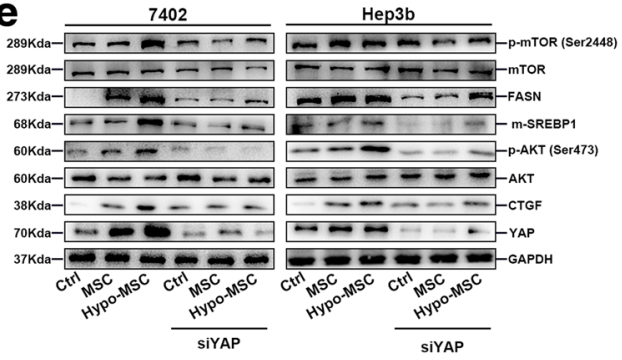

g

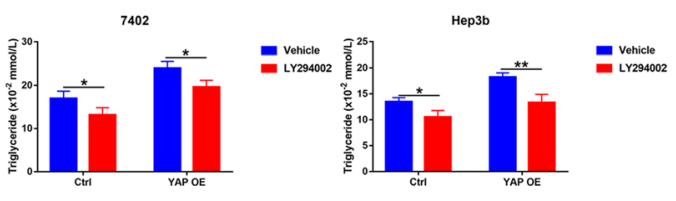

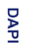

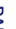

\section{중}

.

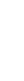


Hypo-MSC-derived $\mathrm{PGE}_{2}$ promotes $\mathrm{HCC}$ via prostaglandin E receptor 4 (EP4)

$\mathrm{PGE}_{2}$ is known to have four types of receptors: EP1-4. Thus, we next investigated the mechanisms of how hypo-MSC-derived $\mathrm{PGE}_{2}$ activated YAP in HCC cells. We found that EP4 was significantly upregulated in hypo-MSC treated HCC cells (Fig. 6a, b), as well as the cAMP response element binding protein (CREB) (Fig. 6c), which has been recently shown to promote YAP activation in HCC [8]. To confirm the role of EP4 in hypo-MSC mediated HCC progression, we used EP4 agonist, Cay10598, to activate EP4 in HCC cells, and found YAP was also activated (Fig. 6d, e). We also knocked down EP4 in HCC cells using siRNA, and found that hypo-MSC was unable to improve HCC cell proliferation (Fig. 6f), and the expression of $Y A P$ and its target genes were suppressed in $\mathrm{HCC}$ cells (Fig. 6g, h). Consistently, exogenous $\mathrm{PGE}_{2}$ increased EP4 expression and failed to activate YAP when EP4 was knocked down (Additional file 8: Figure S7).

Next, we explored the role of EP4 in hypo-MSC induced lipogenesis. Knocking down EP4 significantly reduced the intracellular TG levels, BODIPY staining intensity, and the expression of lipogenic enzymes in HCC cells (Fig. 7a-d). Moreover, the levels of SREBP1, p-AKT and p-mTOR were decreased with EP4 knockdown (Fig. 7b). We also treated the HCC cells with EP4 antagonist GW627368X, and found similar effects on YAP and lipogenesis as EP4 siRNA (Additional file 9: Figure S8). Altogether, our results indicated that hypo-MSC promoted YAP and lipogenesis via $\mathrm{PGE}_{2} / \mathrm{EP} 4$ axis.

\section{Discussion}

As an important part of TME, MSC have been shown to promote cancer progression by numerous studies [1]. Recent studies have also indicated that tumorigenesis heavily relies on the reciprocal interactions between tumor cells and the surrounding stroma $[2,30]$. Therefore, identifying the critical pathway involved in this cross-talk could potentially improve the efficacy of cancer treatment [31, 32]. Hypoxia is an important hallmark of solid tumors, and is associated with tumor progression

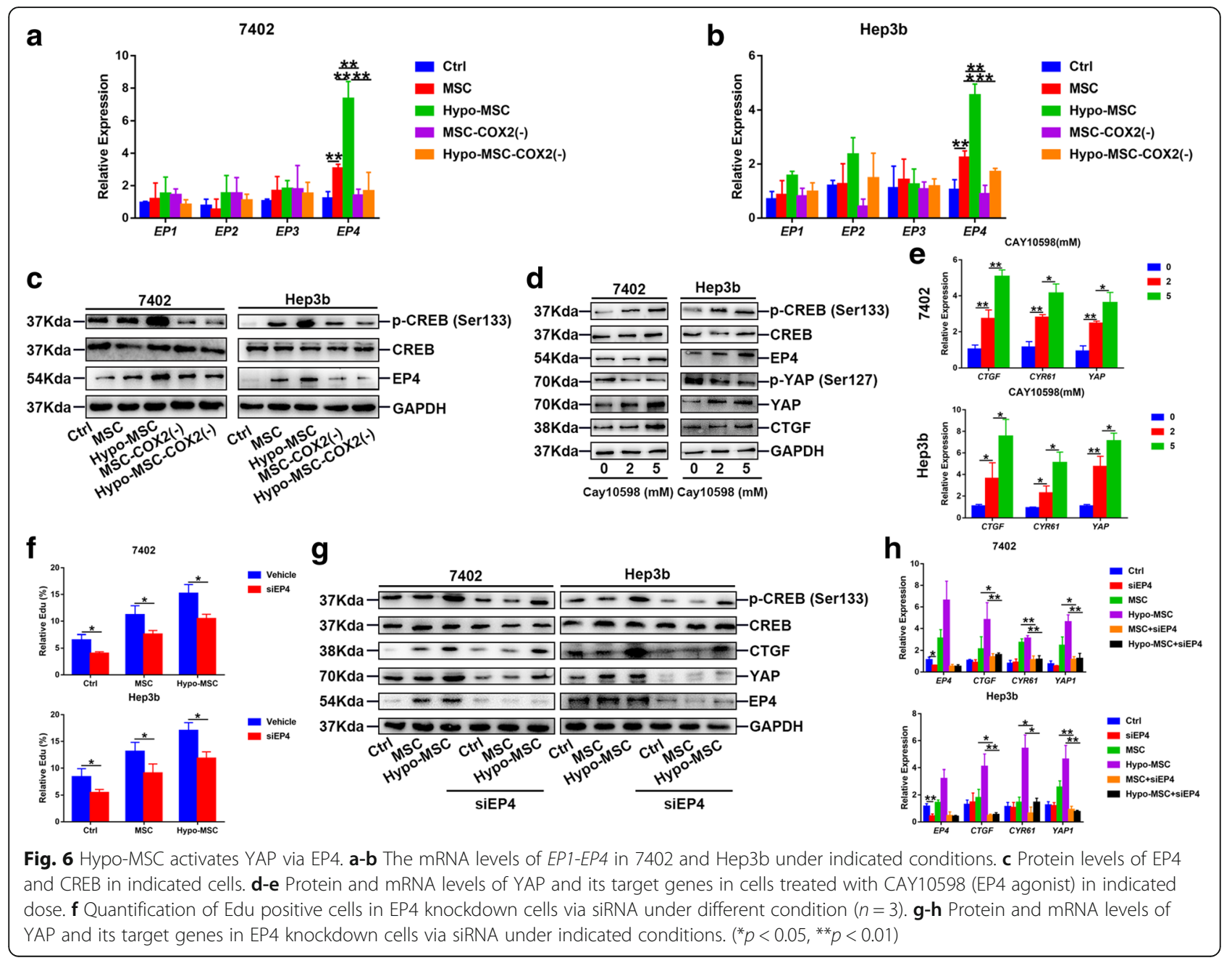


a
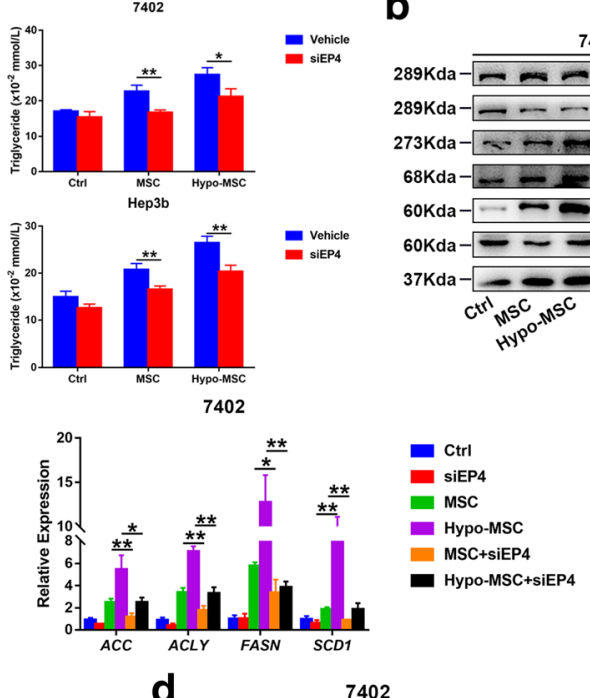

d
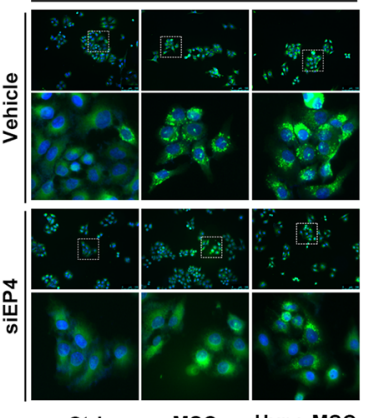

Ctrl

\section{b}
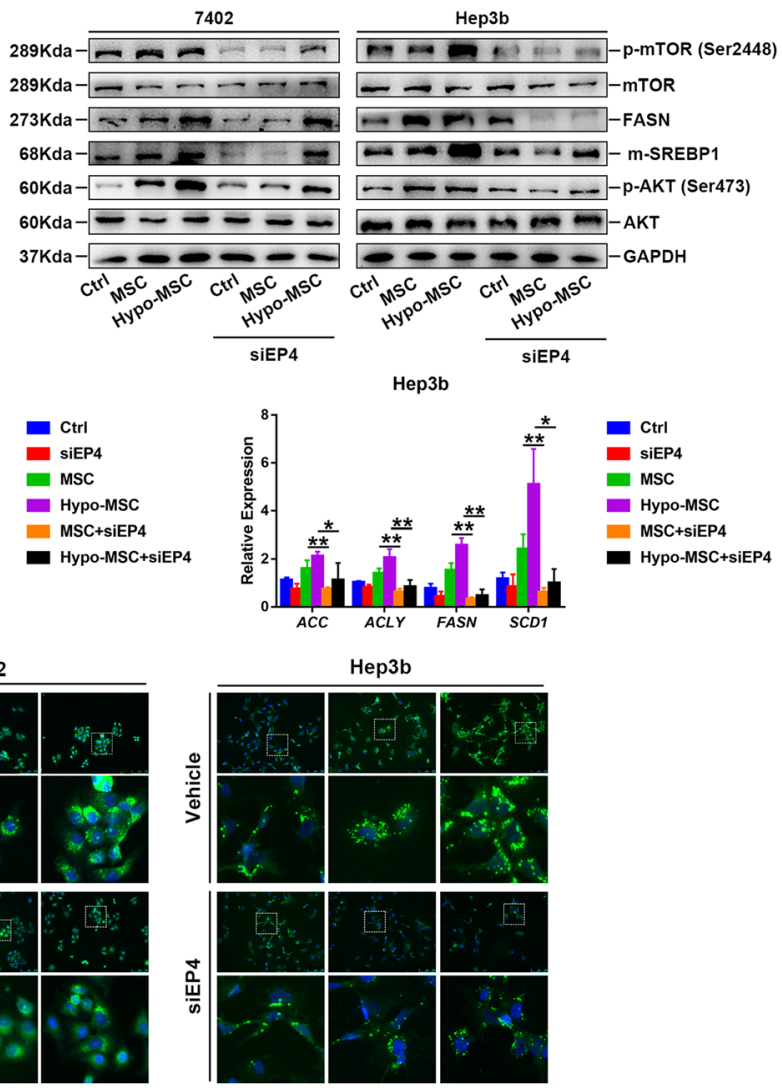

Ctrl

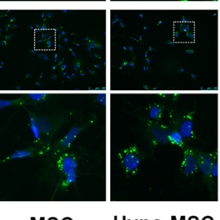

MSC Hypo-MSC

e

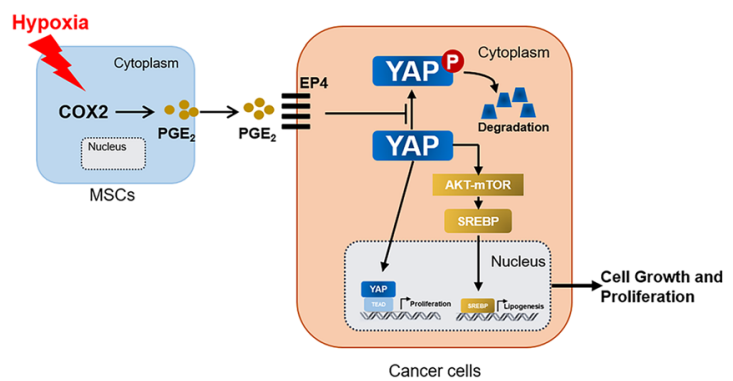

Fig. 7 Hypo-MSC promotes lipogenesis via EP4 mediated YAP activation in cells. a Cellular levels of TG in EP4 knockdown cells under indicated conditions $(n=3)$. b Protein levels of AKT, mTOR and SREBP1 in EP4 knockdown cells under indicated conditions. c The mRNA levels of lipogenic enzymes in EP4 knockdown cells under indicated conditions $(n=3)$. $\mathbf{d}$ The content of neutral lipids in EP4 knockdown cells under indicated conditions. e Proposed model for hypo-MSC on HCC progression. ( ${ }^{*} p<0.05,{ }^{* *} p<0.01$ )

[33]. In this study, we further demonstrated the critical role of hypoxia in MSC mediated HCC progression.

Previous studies have confirmed the formation of paracrine loop between MSC and tumor cells, which resulting in tumor progression and metastasis [34]. Thus, we hypothesized that the cytokines secreted by MSC might be changed under hypoxia condition, leading to increased HCC cell proliferation. COX2 has been shown to induce tumor initiation, progression and angiogenesis in different cancer types. For example, hepatic COX2 overexpression induced spontaneous hepatocellular carcinoma formation in mice [35, 36]. However, most of these studies focused on the role of COX2 in parenchymal cells. Recently, several studies revealed the role of COX2 in noparenchymal cells residing in TME. For example, the myeloid-derived suppressor cells (MDSCs) and macrophages could promote cancer progression through secreting $\mathrm{PGE}_{2}$, a key product of COX2 [37, 38]. Moreover, COX2 expression could be upregulated by hypoxia [20,39], which is consistent with 
our findings. In this study, we found that hypoxia could increase COX2 expression in MSC, which led to enhanced $\mathrm{PGE}_{2}$ secretion and HCC progression. However, knockdown of COX2 did not completely eliminate the effect on $\mathrm{HCC}$ progression under hypoxia condition, indicating that other cytokines in MSC might also be upregulated under hypoxia condition and promote HCC growth.

Although many evidences have pointed out that YAP is a key regulator in many types of solid tumors, the role of YAP activation in HCC has not been fully elucidated. In the present study, we observed increased expression and nuclear accumulation of YAP in HCC cells treated with hypo-MSC. Knockdown of COX2 in MSC suppressed the expression of YAP and impaired the effect of MSC on HCC cell proliferation, even under hypoxia condition. This result is consistent with the previous evidence that $\mathrm{PGE}_{2}$ can activate YAP in colon cancer [40]. Moreover, depletion of YAP significantly impaired the effect of MSC on HCC cell proliferation, demonstrating the importance of YAP in hypo-MSC mediated HCC cell proliferation.

Currently, it has been suggested that YAP is an integrator of metabolic cues and cell growth signals [13]. In HCC, the high mobility group box-1 protein (HMGB1)YAP-dependent aerobic glycolysis played an important role in tumor growth [41]. Also, the effects of YAP on inflammation have also been implicated in NASH progression, indicating that YAP might participate in liver lipid metabolism [42, 43]. Consistent with the previous results, our study demonstrated that hypo-MSC promoted lipogenesis in HCC cells via YAP and SREBP1, which then upregulated the key lipogenic enzymes. Several studies have suggested that YAP regulates AKT signaling components, like PI3K, AKT and PTEN [44-47]; on the other hand, MST1, a Hippo pathway component, inhibited AKT activity in Drosophila [47]. In human liver tumors, increased expression of YAP is associated with high levels of p-AKT [29], and recent study has showed that Hippo pathway prevented hepatic steatosis and liver tumors by suppressing the insulin receptor substrate (IRS)/AKT signaling [43]. Our study confirmed that YAP activated AKT/mTOR signaling, which then activated SREBP1 and promoted lipogenesis.

$\mathrm{PGE}_{2}$ exerts its physiological functions by binding to specific receptors (EP1-4). In this study, we confirmed that hypo-MSC derived $\mathrm{PGE}_{2}$ enhanced cell proliferation via EP4, which activated YAP and the YAP mediated lipogenesis. Consistently, knockdown of EP4 or EP4 antagonists inhibited, while EP4 agonists promoted, the cell proliferation and YAP activation.

\section{Conclusion}

Our work demonstrated that hypo-MSC played a pivotal role in $\mathrm{HCC}$ progression through the COX2/PGE $/ \mathrm{EP}_{2} /$ YAP axis. In particular, our data showed that the YAP activation in HCC cells activated AKT/mTOR/SREBP1 pathway, which then enhanced the lipogenesis and accelerated the growth of HCC cells (Fig. 7e). Thus, our findings characterize the role of cross-talk between MSC and HCC cells in HCC development, providing new insights into the mechanisms underlying the interactions between TME and HCC cells. The newly identified mechanism could potentially serve as a target for HCC therapy.

\section{Additional files}

Additional file 1: Table S1. Antibodies for immunoblots and immunohistochemistry. Table S2. Sequence of primers of qRT-PCR used in experiment. Table S3. Primers of siRNAs. Table S4. Sequence of lentivirus. (DOCX $17 \mathrm{~kb}$ )

Additional file 2: Figure S1. CD90 staining of MSC in xenograft tumors. (DOCX 618 kb)

Additional file 3: Figure S2. Exogenous $\mathrm{PGE}_{2}$ promotes $\mathrm{HCC}$ cell proliferation. (a) The proliferation ability of 7402 and Hep3b treated with $\mathrm{PGE}_{2}$ in indicated dose. (b-c) Representative images and quantification of Edu positive cells in 7402 and Hep3b cells treated with $\mathrm{PGE}_{2}$ in indicated dose $(n=3)$. $\left({ }^{*} p<0.05\right)$. (DOCX $\left.397 \mathrm{~kb}\right)$

Additional file 4: Figure S3. Exogenous $\mathrm{PGE}_{2}$ activates $Y A P$ in $\mathrm{HCC}$ cell lines. (a) Protein levels of YAP and its target CTGF in 7402 and Hep3b cells treated with $P G E_{2}$ in indicated dose. (b-c) Immunofluorescence of YAP in in 7402 and Hep3b cells and quantitative data percentage of cells with nuclear YAP in cells treated with $P G E_{2}$ in indicated dose $(n=3)$. (d) The mRNA levels of YAP and its target genes (CTGF, CYR61) in 7402 and Hep3b cells treated with $P G E_{2}$ in indicated dose. $\left({ }^{*} p<0.05,{ }^{* *} p<0.01\right)$. (DOCX $512 \mathrm{~kb}$ )

Additional file 5: Figure S4. The role of SREBP1 in cell proliferation. (a) Protein levels of SREBP1 in 7402 and Hep3b which transfected with siRNA. (b) The proliferation ability of 7402 and Hep3b after SREBP1 knockdown via siRNA under indicated conditions $(n=3)$. (c) Quantification of Edu positive cells in 7402 and Hep3b cells after SREBP1 knockdown via siRNA under indicated conditions $(n=3)$. (d) The proliferation ability of 7402 and Hep3b after SREBP1 knockdown via siRNA in normal or YAP OE cells $(n=3)$. (e) Quantification of Edu positive cells in 7402 and Hep3b cells after SREBP1 knockdown via siRNA in normal or YAP OE cells $(n=3) .\left({ }^{*} p<0.05,{ }^{* *} p<0.01\right)$. (DOCX $\left.221 \mathrm{~kb}\right)$

Additional file 6: Figure S5. YAP regulates lipogenesis in the presence of SREBP1. (a) Cellular TG levels in YAP OE cells treated with SREBP1 inhibitor, Fatostatin. (b) The mRNA levels of lipogenic enzymes in cells treated with Fatostatin $(n=3)$. (c) The content of neutral lipids in cells treated with Fatostatin. $\left({ }^{*} p<0.05,{ }^{* *} p<0.01\right)$. (DOCX $\left.494 \mathrm{~kb}\right)$

Additional file 7: Figure S6. The interaction between SREBP1 and YAP was examined by CO-IP. (DOCX $91 \mathrm{~kb}$ )

Additional file 8: Figure S7. $\mathrm{PGE}_{2}$ activates YAP via EP4 to promote cell proliferation. (a) the mRNA levels of EP1-EP4 in cells treated with PGE 2 . (b) Expression of EP4 and CREB in cells treated with $P G E_{2}$. (c) Expression of EP4, CREB and YAP in EP4 knockdown cells treated with PGE $E_{2}$. (d) Quantification of Edu positive cells in EP4 knockdown cells treated with $P G E_{2}$. (e) The mRNA levels of YAP and its target genes in EP4 knockdown cells treated with $\left.\mathrm{PGE}_{2} .{ }^{*} p<0.05,{ }^{* *} p<0.01,{ }^{* * *} p<0.001\right)$. (DOCX $432 \mathrm{~kb}$ )

Additional file 9: Figure S8. The role of GW627368X (EP4 inhibitor, EP4i) on hypo-MSC mediated cell proliferation and lipogenesis. (a) Quantification of Edu positive cells in cells treatment of EP4i under indicated conditions. (b) Expression of YAP in cells treatment of EP4i under indicated conditions. (c) The MRNA levels of YAP and its target genes in cells treatment of EP4i under indicated conditions. (d) Expression of AKT, mTOR and SREBP1 in cells treatment of EP4i under indicated conditions. (e) Cellular TG levels in cells treatment of EP4i under indicated conditions. ( $f$ ) The content of neutral lipids in cells treatment of EP4i under indicated conditions. EP4i: EP4 inhibitor. $\left({ }^{*} p<0.05,{ }^{* *} p<0.01\right)$. (DOCX $1210 \mathrm{~kb})$ 


\section{Abbreviations}

ACC1: Acetyl-CoA carboxylase; ACLY: ATP citrate lyase; CCK-8: Cell counting kit-8; CCL5: CC-chemokine ligand 5; COX2: Cyclooxygenase 2; CREB: CAMP response element binding protein; CXCL10: CXC-chemokine ligand 10; EP4: Prostaglandin E receptor 4; FASN: Fatty acid synthase; HCC: Hepatocellular carcinoma; MSC: Mesenchymal stem cell; $\mathrm{PGE}_{2}$ : Prostaglandin $\mathrm{E}_{2} ;$ qRT-PCR: quantitative real-time PCR; SCD1: StearoylCOA desaturase 1; siRNA: small interfering RNA; SREBP: Sterol regulatory elementary binding proteins; TG: Triacylglycerol; TGF $\beta$ : Transforming growth factor- $\beta$; TME: Tumor microenvironment; YAP: Yes-associated protein

\section{Acknowledgements}

Not applicable.

\section{Funding}

This research was supported by grants from the National Natural Science Foundation of China (grant no.81670566 and 81872359).

\section{Availability of data and materials}

All data generated or analyzed during this study are included in this published article (and its supplementary information files).

\section{Authors' contributions}

$Y L, H Z R$ and $Y Z$ performed the experimental work. LCS, FJY and YHZ participated in the experiments. XLS conceived the study and participated in its design and coordination. The manuscript was written by $\mathrm{YL}$ and $\mathrm{YZ}$. All authors read and approved the final manuscript.

\section{Ethics approval and consent to participate}

All animal experiments were performed with the approval of Ethics Committee for Animal Experimentation of the Affiliated Drum Tower Hospital of Nanjing University Medical School.

\section{Consent for publication}

Not applicable.

\section{Competing interests}

The authors declare that they have no competing interests.

\section{Publisher's Note}

Springer Nature remains neutral with regard to jurisdictional claims in published maps and institutional affiliations.

\section{Received: 19 December 2018 Accepted: 8 May 2019}

Published online: 29 May 2019

\section{References}

1. Shi Y, Du L, Lin L, Wang Y. Tumour-associated mesenchymal stem/stromal cells: emerging therapeutic targets. Nat Rev Drug Discov. 2016;16(1):35-52.

2. Quail DF, Joyce JA. Microenvironmental regulation of tumor progression and metastasis. Nat Med. 2013;19(11):1423-37.

3. Gonzalez ME, Martin EE, Anwar T, Arellano-Garcia C, Medhora N, Lama A, et al. Mesenchymal stem cell-induced DDR2 mediates stromal-breast Cancer interactions and metastasis growth. Cell Rep. 2017;18(5):1215-28.

4. Triner D, Shah YM. Hypoxia-inducible factors: a central link between inflammation and cancer. J Clin Invest. 2016;126(10):3689-98.

5. Totaro A, Panciera T, Piccolo S. YAP/TAZ upstream signals and downstream responses. Nat Cell Biol. 2018;20(8):888-99.

6. Patel $\mathrm{SH}$, Camargo FD, Yimlamai D. Hippo signaling in the liver regulates organ size, cell fate, and carcinogenesis. Gastroenterology. 2017;152(3):533-45.

7. Perra A, Kowalik MA, Ghiso E, Ledda-Columbano GM, Di Tommaso L, Angioni MM, et al. YAP activation is an early event and a potential therapeutic target in liver cancer development. J Hepatol. 2014;61(5):1088-96.

8. Wang J, Ma L, Weng W, Qiao Y, Zhang Y, He J, et al. Mutual interaction between YAP and CREB promotes tumorigenesis in liver cancer. Hepatology. 2013;58(3):1011-20.

9. Yimlamai D, Fowl BH, Camargo FD. Emerging evidence on the role of the hippo/YAP pathway in liver physiology and cancer. J Hepatol. 2015;63(6): 1491-501.

10. Rohrig F, Schulze A. The multifaceted roles of fatty acid synthesis in cancer. Nat Rev Cancer. 2016;16(11):732-49.
11. Currie E, Schulze A, Zechner R, Walther TC, Farese RV Jr. Cellular fatty acid metabolism and cancer. Cell Metab. 2013;18(2):153-61.

12. Calvisi DF, Wang C, Ho C, Ladu S, Lee SA, Mattu S, et al. Increased lipogenesis, induced by AKT-mTORC1-RPS6 signaling, promotes development of human hepatocellular carcinoma. Gastroenterology. 2011; 140(3):1071-83.

13. Koo JH, Guan KL. Interplay between YAP/TAZ and metabolism. Cell Metab. 2018;28(2):196-206.

14. Ardestani A, Lupse B, Maedler K. Hippo signaling: key emerging pathway in cellular and whole-body metabolism. Trends Endocrinol Metab. 2018;29(7): 492-509.

15. Liu Y, Ren H, Wang J, Yang F, Li J, Zhou Y, et al. Prostaglandin E2 secreted by mesenchymal stem cells protects against acute liver failure via enhancing hepatocyte proliferation. FASEB J. 2019;33(2):2514-25.

16. Cao MQ, You AB, Zhu XD, Zhang W, Zhang YY, Zhang SZ, et al. miR-182-5p promotes hepatocellular carcinoma progression by repressing FOXO3a. J Hematol Oncol. 2018:11(1):12.

17. Yin F, Sharen G, Yuan F, Peng Y, Chen R, Zhou X, et al. TIP30 regulates lipid metabolism in hepatocellular carcinoma by regulating SREBP1 through the Akt/mTOR signaling pathway. Oncogenesis. 2017;6(6):e347.

18. Xue X, Shah YM. Hypoxia-inducible factor-2alpha is essential in activating the COX2/mPGES-1/PGE2 signaling axis in colon cancer. Carcinogenesis. 2013;34(1):163-9.

19. Zhao L, Wu Y, Xu Z, Wang H, Zhao Z, Li Y, et al. Involvement of COX-2/PGE2 signalling in hypoxia-induced angiogenic response in endothelial cells. J Cell Mol Med. 2012;16(8):1840-55.

20. Lee JJ, Natsuizaka M, Ohashi S, Wong GS, Takaoka M, Michaylira CZ, et al. Hypoxia activates the cyclooxygenase-2-prostaglandin E synthase axis. Carcinogenesis. 2010;31(3):427-34.

21. Nakanishi M, Rosenberg DW. Multifaceted roles of PGE2 in inflammation and cancer. Semin Immunopathol. 2013;35(2):123-37.

22. Ooki A, Rodriguez MDC, Pena L, Marchionni W, Dinalankara A, Begum NMH, et al. YAP1 and COX2 coordinately regulate urothelial Cancer stem-like cells. Cancer Res. 2018;78(1):168-81.

23. Cheng JC, Chang HM, Liu PP, Leung PC. Sphingosine-1-phosphate induces COX-2 expression and PGE2 production in human granulosa cells through a S1P1/3-mediated YAP signaling. Cell Signal. 2016;28(6):643-51.

24. Xu G, Wang Y, Li W, Cao Y, Xu J, Hu Z, et al. COX-2 forms regulatory loop with YAP to promote proliferation and tumorigenesis of hepatocellular carcinoma cells. Neoplasia. 2018;20(4):324-34.

25. Shimano H, Sato R. SREBP-regulated lipid metabolism: convergent physiology - divergent pathophysiology. Nat Rev Endocrinol. 2017; 13(12):710-30.

26. Li C, Yang W, Zhang J, Zheng X, Yao Y, Tu K, et al. SREBP-1 has a prognostic role and contributes to invasion and metastasis in human hepatocellular carcinoma. Int J Mol Sci. 2014;15(5):7124-38.

27. Aylon Y, Gershoni A, Rotkopf R, Biton IE, Porat Z, Koh AP, et al. The LATS2 tumor suppressor inhibits SREBP and suppresses hepatic cholesterol accumulation. Genes Dev. 2016:30(7):786-97.

28. Saxton RA, Sabatini DM. mTOR signaling in growth, metabolism, and disease. Cell. 2017:168(6):960-76.

29. Sohn BH, Shim JJ, Kim SB, Jang KY, Kim SM, Kim JH, et al. Inactivation of hippo pathway is significantly associated with poor prognosis in hepatocellular carcinoma. Clin Cancer Res. 2016;22(5):1256-64.

30. Taddei ML, Giannoni E, Comito G, Chiarugi P. Microenvironment and tumor cell plasticity: an easy way out. Cancer Lett. 2013;341(1):80-96.

31. Valkenburg $\mathrm{KC}$, de Groot $\mathrm{AE}$, Pienta KJ. Targeting the tumour stroma to improve cancer therapy. Nat Rev Clin Oncol. 2018;15(6):366-81.

32. Klemm F, Joyce JA. Microenvironmental regulation of therapeutic response in cancer. Trends Cell Biol. 2015;25(4):198-213.

33. Schito L, Semenza GL. Hypoxia-inducible factors: master regulators of Cancer progression. Trends Cancer. 2016;2(12):758-70.

34. Sun Z, Wang S, Zhao RC. The roles of mesenchymal stem cells in tumor inflammatory microenvironment. J Hematol Oncol. 2014;7:14.

35. Chen H, Cai W, Chu ESH, Tang J, Wong CC, Wong SH, et al. Hepatic cyclooxygenase-2 overexpression induced spontaneous hepatocellular carcinoma formation in mice. Oncogene. 2017;36(31):4415-26.

36. Ko CJ, Lan SW, Lu YC, Cheng TS, Lai PF, Tsai CH, et al. Inhibition of cyclooxygenase-2-mediated matriptase activation contributes to the suppression of prostate cancer cell motility and metastasis. Oncogene. 2017; 36(32):4597-609. 
37. Prima V, Kaliberova LN, Kaliberov S, Curiel DT, Kusmartsev S. COX2/mPGES1/ PGE2 pathway regulates PD-L1 expression in tumor-associated macrophages and myeloid-derived suppressor cells. Proc Natl Acad Sci U S A. 2017:114(5):1117-22.

38. Yan G, Zhao H, Zhang Q, Zhou Y, Wu L, Lei J, et al. A RIPK3-PGE2 circuit mediates myeloid-derived suppressor cell-potentiated colorectal carcinogenesis. Cancer Res. 2018;78(19):5586-99.

39. Samarajeewa NU, Yang F, Docanto MM, Sakurai M, McNamara KM, Sasano $\mathrm{H}$, et al. HIF-1alpha stimulates aromatase expression driven by prostaglandin E2 in breast adipose stroma. Breast Cancer Res. 2013;15(2):R30.

40. Kim HB, Kim M, Park YS, Park I, Kim T, Yang SY, et al. Prostaglandin E2 activates YAP and a positive-signaling loop to promote Colon regeneration after colitis but also carcinogenesis in mice. Gastroenterology. 2017;152(3): 616-30.

41. Chen R, Zhu S, Fan XG, Wang H, Lotze MT, Zeh HJ 3rd, et al. High mobility group protein B1 controls liver cancer initiation through yes-associated protein -dependent aerobic glycolysis. Hepatology. 2018;67(5):1823-41.

42. Wang X, Zheng Z, Caviglia JM, Corey KE, Herfel TM, Cai B, et al. Hepatocyte TAZMWTR1 promotes inflammation and fibrosis in nonalcoholic steatohepatitis. Cell Metab. 2016;24(6):848-62.

43. Jeong SH, Kim HB, Kim MC, Lee JM, Lee JH, Kim JH, et al. Hippo-mediated suppression of IRS2/AKT signaling prevents hepatic steatosis and liver cancer. J Clin Invest. 2018;128(3):1010-25.

44. Lin Z, Zhou P, von Gise A, Gu F, Ma Q, Chen J, et al. Pi3kcb links hippo-YAP and PI3K-AKT signaling pathways to promote cardiomyocyte proliferation and survival. Circ Res. 2015:116(1):35-45.

45. Cinar B, Fang PK, Lutchman M, Di Vizio D, Adam RM, Pavlova N, et al. The pro-apoptotic kinase Mst1 and its caspase cleavage products are direct inhibitors of Akt1. EMBO J. 2007;26(21):4523-34.

46. Tumaneng K, Schlegelmilch K, Russell RC, Yimlamai D, Basnet H, Mahadevan $\mathrm{N}$, et al. YAP mediates crosstalk between the hippo and PI (3) K-TOR pathways by suppressing PTEN via miR-29. Nat Cell Biol. 2012;14(12):1322-9.

47. Ye X, Deng Y, Lai ZC. Akt is negatively regulated by hippo signaling for growth inhibition in Drosophila. Dev Biol. 2012;369(1):115-23.

Ready to submit your research? Choose BMC and benefit from:

- fast, convenient online submission

- thorough peer review by experienced researchers in your field

- rapid publication on acceptance

- support for research data, including large and complex data types

- gold Open Access which fosters wider collaboration and increased citations

- maximum visibility for your research: over $100 \mathrm{M}$ website views per year

At $\mathrm{BMC}$, research is always in progress.

Learn more biomedcentral.com/submissions 